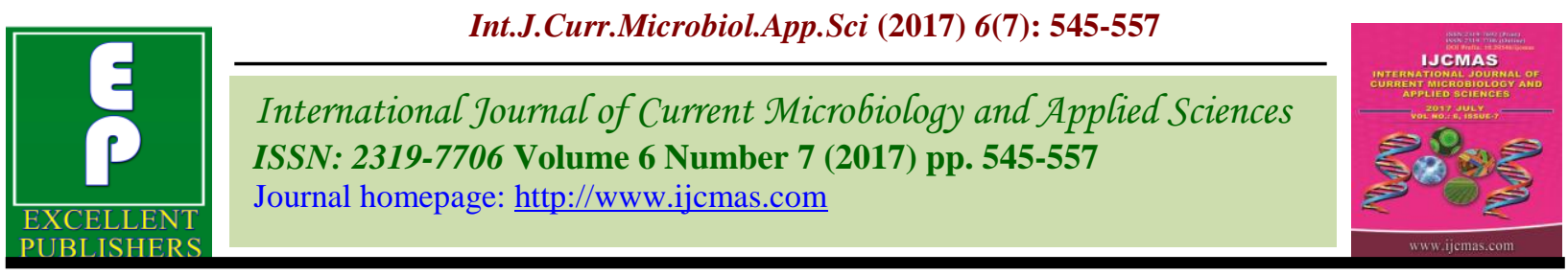

Original Research Article

https://doi.org/10.20546/ijcmas.2017.607.066

\title{
Plant Regeneration from Hypocotyl Explants in Capsicum (Capsicum annuum L.)
}

\author{
Vivek Hegde $^{1 *}$, P.S. Partap ${ }^{1}$ and R.C. Yadav ${ }^{2}$ \\ ${ }^{1}$ Department of Vegetable Science, ${ }^{2}$ Department of Molecular Biology and Biotechnology, CCS \\ Haryana Agricultural University, Hisar-125 004, Haryana, India \\ *Corresponding author
}

\section{A B S T R A C T}

\begin{tabular}{|l|}
\hline Ke y w or d s \\
In vitro \\
regeneration, \\
Hypocotyl, \\
Capsicum, \\
Tissue culture. \\
\hline Article Info \\
\hline Accepted: \\
04 June 2017 \\
Available Online: \\
10 July 2017 \\
\hline \hline
\end{tabular}

Development of an efficient plant regeneration protocol is the pre-requisite for crop improvement through biotechnological approach. Hence, the in vitro regeneration was achieved from hypocotyl explants. The explants were taken from aseptically raised seedlings of popular Capsicum $\mathrm{F}_{1}$ hybrids Bharat and Indra. Seeds of both hybrids were soaked in distilled water along with $\mathrm{GA}_{3}$ at $2 \mathrm{mg} / \mathrm{l}$ to get optimum germination and decontaminated prior to in vitro sowing on half-strength MS medium. Tissue culture responses to morphogenesis varied with the genotypes and combinations of growth regulators used. Per cent regeneration (44.44\%), number of shoots per explants (2.11) and per cent elongation $(66.85 \%)$ was maximum in hybrid Indra from hypocotyl explants in medium supplemented with zeatin at $7.5 \mathrm{mg} / \mathrm{l}$ along with $\mathrm{GA}_{3}$ at $2.0 \mathrm{mg} / \mathrm{l}$. Cent per cent rooting, optimum number of roots (27.56 and 23.65, respectively) and root length $(4.94 \mathrm{~cm}$ and $7.71 \mathrm{~cm}$, respectively) were observed when regenerated shoots were cultured on MS media supplemented with $0.5 \mathrm{mg} / \mathrm{l} \mathrm{IBA}$ in both the hybrids Indra and Bharat. The regenerated plantlets were hardened in pots filled with a sterile mixture of coco-peat and vermiculite (1:1) and thereafter transferred to pots containing soil. The Survival percentage was $85.7 \%$ to $92.3 \%$ in hybrids Bharat and Indra, respectively.

\section{Introduction}

Capsicum (Capsicum annuum L.) is one of the most important solanaceous vegetable crops in the world and commonly known as sweet pepper, bell pepper or pepper. This essential kitchen item, occupying a very important place in the Indian diet. It is consumed daily in one or the other form as a vegetable, spice, adding colour, flavour, pungency and piquancy to various foodstuffs. It is an excellent source of several vitamins like A, B-complex, C, E and also rich in minerals like manganese, molybdenum and potassium (Simonne et al., 1997).

In spite of different varieties of capsicum found all over the world, the susceptibility of these genotypes to the biotic and abiotic stresses including the fungi, bacteria, viruses and insect pests and the extremes of moisture, temperature, light, nutrients and $\mathrm{pH}$ has extremely restricted their quality and yield potential (Venkataiah et al., 2003; Suzuki and Mori, 2003; Ochoa and Ramirez, 2001 and Egea et al., 2002). Although, capsicum production, quality and use have been upgraded remarkably due to better agricultural practices combined with improved varieties through conventional plant 
breeding. But the restricted gene pool has placed a limit to further improvement in capsicum.

Hence, to boost the crop improvement programs, the plant biotechnology covering tissue culture, in vitro selection, in vitro mutation and genetic engineering are becoming a functional tool of classical plant breeding. Standardisation of protocol for in vitro plant regeneration from protoplast, cells, tissues and organ cultures is the fundamental process for biotechnology applications in plant propagation and genetic improvement. This would serve the main purposes of mass multiplication of elite plants, micropropagation of pollen-derived haploid plants, maintenance of male sterile lines, in vitro selection of plants against abiotic and biotic stresses, in vitro induction of useful mutation and the genetic transformation.

The factors like genotype, explants, growth regulators and their concentrations etc., are influence the in vitro regeneration responses of crop plants (Dabauza and Pena, 2001). The genetic manipulation of Capsicum has been unsuccessful because of its highly recalcitrant and high genotype specificity in in vitro plant regeneration, are the large bottleneck to transferring the desired genes (Sharma et al., 2017). Hence, regeneration response of hypocotyl explants from popular capsicum $\mathrm{F}_{1}$ hybrids, Bharat and Indra were studied and presented in this paper.

\section{Materials and Methods}

Among the popular $F_{1}$ hybrids of capsicum, the Bharat and Indra were chosen for the present study. The seeds were washed in running tap water followed by tween-20 and soaked at room temperature in distilled water with $2 \mathrm{mg} / \mathrm{l} \mathrm{GA}_{3}$ for 2 days. Subsequently, the soaked seeds were surface sterilized with 0.1 per cent mercuric chloride $\left(\mathrm{HgCl}_{2}\right)$ for 5 to 6 minutes under laminar-airflow cabinet. Thereafter, seeds were washed 3 to 4 times with autoclaved sterile distilled water to remove the traces of $\mathrm{HgCl}_{2}$. Surface sterilized seeds were inoculated on half strength MS basal medium and were incubated under $16 \mathrm{~h}$ light and $8 \mathrm{~h}$ dark photoperiod at $25 \pm 2{ }^{\circ} \mathrm{C}$ for raising the seedlings. The hypocotyl explants for regeneration were taken from these in vitro grown, 5-7 day old seedlings. The 0.4 to $0.6 \mathrm{~cm}$ hypocotyl explants were inoculated horizontally (Plate 1A) on MS medium containing three per cent sucrose, 0.8 per cent agar supplemented with different concentrations and combinations of growth regulators. After inoculation of hypocotyl explants in Petri-dishes containing MS media with different treatments were properly sealed with the parafilm strips to protect from contamination.

Cultures were incubated in a culture room at $25 \pm 2{ }^{\circ} \mathrm{C}$ under photoperiod of $16 \mathrm{~h}$ light and $8 \mathrm{~h}$ dark. The well-elongated shoots were obtained from shooting medium were separated and transferred to MS medium supplemented with auxins IBA and NAA of different concentrations $(0.0,0.5,1.0 \mathrm{mg} / \mathrm{l})$ for root induction. These cultures were incubated at $25 \pm 2{ }^{\circ} \mathrm{C}$ under $16 \mathrm{~h}$ light and $8 \mathrm{~h}$ dark photoperiod for proper rooting.

Plantlets grown in vitro were removed from the culture bottles without damaging their root systems. To remove the traces of medium sticking to the roots were washed properly under running tap water. Then the well-rooted plantlets were transferred to pots containing the sterile mixture of cocopeat and vermiculite (1:1) for hardening and the nutrients were supplied at five day intervals through the application of liquid MS basal salts without sucrose. To maintain humidity around the potted plants, 
each pot was covered with a polythene bag. Within 6-7 days, the polythene bags were removed and after 14 days of acclimatization under poly-house, plantlets were transferred to big size pot containing soil, sand and farmyard manure at 1:1:1 ratio.

The per cent regeneration, elongated shoots per explants, per cent shoot elongation, per cent root formation, roots per shoot, root length and per cent survivals of regenerated plants in soil were assessed. The mean and standard errors were worked out from triplicate data obtained from various treatments. The per cent data transformed using angular transformation and analyzed following Completely Randomized Design (CRD).

\section{Results and Discussion}

In the present experiment, in vitro regeneration of two capsicum hybrids namely Bharat and Indra were tested. The in vitro germination of capsicum is very slow and uneven germination was observed (Vivek Hegde et al., 2017). Watkins and Cantlife (1983) and Watkins et al., (1985) found that non-starchy endospermic tissues enclosing radical tips might act as a mechanical barrier to the growing embryo and imparted to slow and erratic germination of capsicum seeds. In order to enhance seed germination, seed soaked in distilled water along with $\mathrm{GA}_{3}$ at 2 $\mathrm{mg} / \mathrm{l}$ for two days and sown in vitro to get sterile seedlings.

Application of $\mathrm{GA}_{3}$ might weaken the nonstarchy endospermic tissues which is enclosing radical tips and triggered the germination. Watkins et al., (1985) and Groot and Karssen (1987) reported that in seeds prior to germination, the enzyme activity mediated by $\mathrm{GA}_{3}$ might take part in weakening process of endosperms. Similarly,
Andreoli and Khan (1999) described that $\mathrm{GA}_{3}$ induced enzymes efficiently digest the endosperm cells and improves the seed germination in capsicum.

The hypocotyl explants were obtained from the in vitro grown, 10-12 day old seedlings of both Capsicum hybrids (Bharat and Indra) were utilized in plant regeneration experiment. These explants were observed for plant regeneration response on MS medium containing various concentrations and combinations of growth regulators (Plate 1A).

Genotype is one of the important factor that influence the organogenic response of in vitro plant cultures. The existence of strong genotype specificity in the regeneration capacity of the different cultivar represents an important limiting factor that makes development of a specific regeneration protocols are required for each cultivar. Per cent regeneration, number of elongated shoots per explants and per cent elongation was maximum in hybrid Indra as compared to Bharat, on MS medium supplemented with $7.5 \mathrm{mg} / \mathrm{l}$ zeatin along with $2.0 \mathrm{mg} / 1 \mathrm{GA}_{3}, 7.5$ $\mathrm{mg} / \mathrm{l}$ kinetin along with $2.0 \mathrm{mg} / \mathrm{l} \mathrm{GA}_{3}$ and BAP at $7.5 \mathrm{mg} / \mathrm{l}$ along with $2.0 \mathrm{mg} / \mathrm{GA}_{3}$ (Tables 1, 2 and 4). But, hybrid Bharat responded better than Indra on MS medium containing $5.0 \mathrm{mg} / \mathrm{l} \mathrm{TDZ}$ along with $2.0 \mathrm{mg} / \mathrm{l}$ $\mathrm{GA}_{3}$ (Table 3). The browning of explants was observed on MS medium supplemented with TDZ exceeding $7.5 \mathrm{mg} / \mathrm{l}$. Recent studies have also supported the influence of genotype on organogenesis, for example, Mathew (2002) observed cv. Byadagi Dabbi responded better in in vitro as compared to Arka Lohit. Venkataiah et al., (2003) reported the response depended upon the genotype specifically in TDZ mediated organogenesis in 10 pepper cultivars. Valadez et al., (2009) developed separate in vitro regeneration protocol for four different chilli genotypes. Kumar et al., (2012) observed variable degree 
of regeneration in red pepper cultivars. Marta and Pawel (2015) observed similar organogenesis response in the 3 genotypes of
C. annuиm $\mathrm{L}$. and it was considerably lower in an interspecific hybrid (C. frutescens $\mathrm{L} . \times$ C. annuum).

Plate.1 (A) Cultured hypocotyl explants, (B) Multiple shoots induction from Hypocotyl explants, (C) Multiple shoot elongation

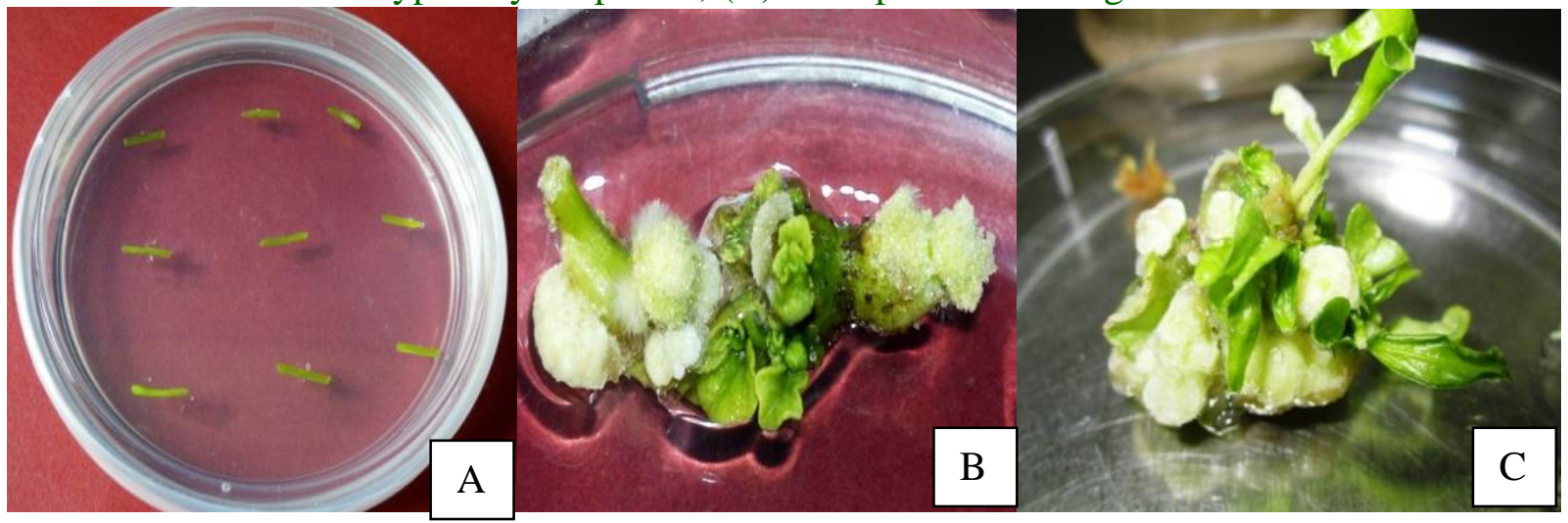

Plate.2 Rooting of in vitro raised shoots; (A) IBA $0.5 \mathrm{mg} / \mathrm{l}$, (B) IBA $1.0 \mathrm{mg} / \mathrm{l}$, (C) NAA $0.5 \mathrm{mg} / \mathrm{l}$ and (D) NAA $1.0 \mathrm{mg} / \mathrm{l}$

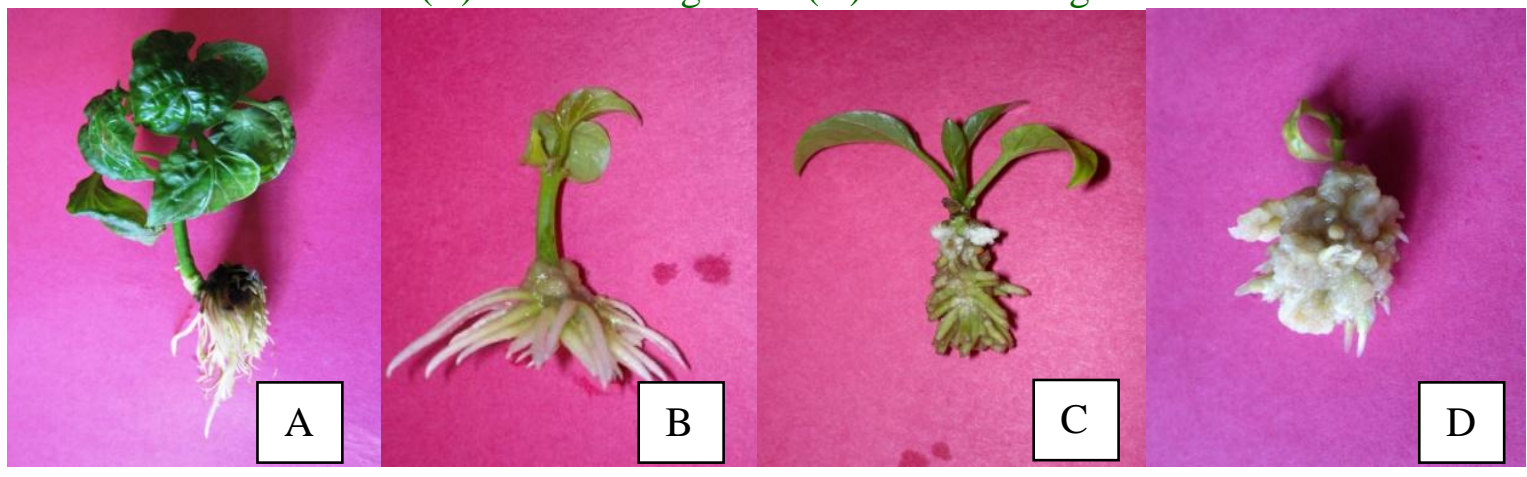

Plate.3 (A) Initial hardening of regenerated plantlets, (B) Regenerated hardened plants

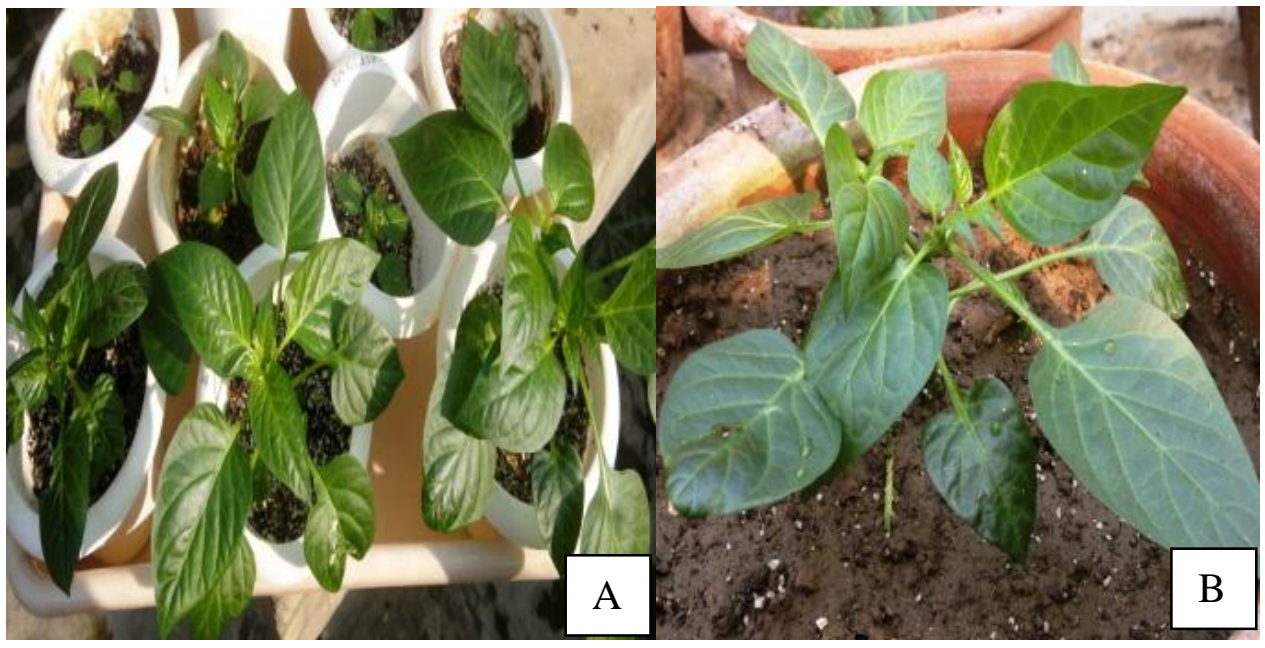


Table.1 Effect of MS medium with $\mathrm{BAP} \pm \mathrm{NAA} / \mathrm{GA}_{3}$ on regeneration of hypocotyl explants of Capsicum hybrids Bharat and Indra

\begin{tabular}{|c|c|c|c|c|c|c|c|c|}
\hline \multirow{2}{*}{\multicolumn{3}{|c|}{$\begin{array}{c}\mathrm{MS}+\text { growth } \\
\text { regulators }(\mathrm{mg} / \mathrm{l})\end{array}$}} & \multicolumn{3}{|c|}{ Bharat } & \multicolumn{3}{|c|}{ Indra } \\
\hline & & & \multirow{2}{*}{$\begin{array}{c}\text { Per cent } \\
\text { Regeneration }\end{array}$} & \multirow{2}{*}{$\begin{array}{c}\text { Elongated shoots } \\
\text { per explant }\end{array}$} & \multirow{2}{*}{$\begin{array}{c}\text { Per cent shoot } \\
\text { elongation }\end{array}$} & \multirow{2}{*}{$\begin{array}{c}\text { Per cent } \\
\text { Regeneration }\end{array}$} & \multirow{2}{*}{$\begin{array}{c}\text { Elongated shoots } \\
\text { per explant }\end{array}$} & \multirow{2}{*}{$\begin{array}{l}\text { Per cent shoo } \\
\text { elongation }\end{array}$} \\
\hline BAP & NAA & $\mathbf{G A}_{3}$ & & & & & & \\
\hline 2.5 & 0.0 & 0.0 & $\begin{array}{c}0.00 \\
(0.0 \pm 0.0)\end{array}$ & $\begin{array}{c}0.00 \\
(1.0 \pm 0.0)\end{array}$ & $\begin{array}{c}0.00 \\
(0.0 \pm 0.0)\end{array}$ & $\begin{array}{c}0.00 \\
(0.0 \pm 0.0)\end{array}$ & $\begin{array}{c}0.00 \\
(1.0 \pm 0.0)\end{array}$ & $\begin{array}{c}0.00 \\
(0.0 \pm 0.0)\end{array}$ \\
\hline 2.5 & 0.2 & 0.0 & $\begin{array}{c}0.00 \\
(0.0 \pm 0.0)\end{array}$ & $\begin{array}{c}0.00 \\
(1.0 \pm 0.0)\end{array}$ & $\begin{array}{c}0.00 \\
(0.0 \pm 0.0)\end{array}$ & $\begin{array}{c}0.00 \\
(0.0 \pm 0.0)\end{array}$ & $\begin{array}{c}0.00 \\
(1.0 \pm 0.0)\end{array}$ & $\begin{array}{c}0.00 \\
(0.0 \pm 0.0)\end{array}$ \\
\hline 2.5 & 0.0 & 2.0 & $\begin{array}{c}0.00 \\
(0.0 \pm 0.0)\end{array}$ & $\begin{array}{c}0.00 \\
(1.0 \pm 0.0)\end{array}$ & $\begin{array}{c}0.00 \\
(0.0 \pm 0.0)\end{array}$ & $\begin{array}{c}0.00 \\
(0.0 \pm 0.0)\end{array}$ & $\begin{array}{c}0.00 \\
(1.0 \pm 0.0)\end{array}$ & $\begin{array}{c}0.00 \\
(0.0 \pm 0.0)\end{array}$ \\
\hline 5.0 & 0.0 & 0.0 & $\begin{array}{c}12.35 \\
(20.5 \pm 1.1)\end{array}$ & $\begin{array}{c}0.00 \\
(1.0 \pm 0.0)\end{array}$ & $\begin{array}{c}16.67 \\
(24.1 \pm 0.0)\end{array}$ & $\begin{array}{c}16.05 \\
(23.6 \pm 1.0)\end{array}$ & $\begin{array}{c}0.00 \\
(1.0 \pm 0.0)\end{array}$ & $\begin{array}{c}0.00 \\
(0.0 \pm 0.0)\end{array}$ \\
\hline 5.0 & 0.2 & 0.0 & $\begin{array}{c}4.94 \\
(12.7 \pm 1.6)\end{array}$ & $\begin{array}{c}0.00 \\
(1.0 \pm 0.0)\end{array}$ & $\begin{array}{c}0.00 \\
(0.0 \pm 0.0)\end{array}$ & $\begin{array}{c}4.94 \\
(12.7 \pm 1.6)\end{array}$ & $\begin{array}{c}0.00 \\
(1.0 \pm 0.0)\end{array}$ & $\begin{array}{c}0.00 \\
(0.0 \pm 0.0)\end{array}$ \\
\hline 5.0 & 0.0 & 2.0 & $\begin{array}{c}12.35 \\
(20.5 \pm 1.1)\end{array}$ & $\begin{array}{c}0.44 \\
(1.2 \pm 0.1)\end{array}$ & $\begin{array}{c}38.89 \\
(38.5 \pm 3.3)\end{array}$ & $\begin{array}{c}16.05 \\
(23.6 \pm 0.0)\end{array}$ & $\begin{array}{c}0.33 \\
(1.2 \pm 0.0)\end{array}$ & $\begin{array}{c}38.89 \\
(38.5 \pm 2.3)\end{array}$ \\
\hline 7.5 & 0.0 & 0.0 & $\begin{array}{c}23.46 \\
(28.9 \pm 0.8)\end{array}$ & $\begin{array}{c}0.89 \\
(1.4 \pm 0.0)\end{array}$ & $\begin{array}{c}44.44 \\
(41.8 \pm 0.0)\end{array}$ & $\begin{array}{c}30.86 \\
(33.7 \pm 0.8)\end{array}$ & $\begin{array}{c}0.89 \\
(1.4 \pm 0.1)\end{array}$ & $\begin{array}{c}39.81 \\
(39.1 \pm 0.5)\end{array}$ \\
\hline 7.5 & 0.2 & 0.0 & $\begin{array}{c}9.88 \\
(18.2 \pm 1.2)\end{array}$ & $\begin{array}{c}0.33 \\
(1.2 \pm 0.0)\end{array}$ & $\begin{array}{c}33.33 \\
(35.3 \pm 0.0)\end{array}$ & $\begin{array}{c}12.35 \\
(20.5 \pm 1.1)\end{array}$ & $\begin{array}{c}0.11 \\
(1.1 \pm 0.1)\end{array}$ & $\begin{array}{c}18.52 \\
(25.4 \pm 1.3)\end{array}$ \\
\hline 7.5 & 0.0 & 2.0 & $\begin{array}{c}23.46 \\
(28.9 \pm 0.8) \\
\end{array}$ & $\begin{array}{c}1.33 \\
(1.5 \pm 0.1)\end{array}$ & $\begin{array}{c}55.56 \\
(48.2 \pm 0.0) \\
\end{array}$ & $\begin{array}{c}30.86 \\
(33.7 \pm 0.8) \\
\end{array}$ & $\begin{array}{c}1.56 \\
(1.6 \pm 0.1)\end{array}$ & $\begin{array}{c}55.56 \\
(48.2 \pm 0.0) \\
\end{array}$ \\
\hline 10.0 & 0.0 & 0.0 & $\begin{array}{c}24.69 \\
(29.8 \pm 0.8)\end{array}$ & $\begin{array}{c}0.89 \\
(1.4 \pm 0.0)\end{array}$ & $\begin{array}{c}46.30 \\
(42.9 \pm 1.1)\end{array}$ & $\begin{array}{c}29.63 \\
(33.0 \pm 0.0)\end{array}$ & $\begin{array}{c}0.78 \\
(1.3 \pm 0.0)\end{array}$ & $\begin{array}{c}38.89 \\
(38.6 \pm 0.0)\end{array}$ \\
\hline 10.0 & 0.2 & 0.0 & $\begin{array}{c}11.11 \\
(19.5 \pm 0.0)\end{array}$ & $\begin{array}{c}0.33 \\
(1.2 \pm 0.0) \\
\end{array}$ & $\begin{array}{c}33.33 \\
(35.3 \pm 0.0) \\
\end{array}$ & $\begin{array}{c}12.35 \\
(20.5 \pm 1.1) \\
\end{array}$ & $\begin{array}{c}0.11 \\
(1.1 \pm 0.1)\end{array}$ & $\begin{array}{c}18.52 \\
(25.2 \pm 2.9)\end{array}$ \\
\hline 10.0 & 0.0 & 2.0 & $\begin{array}{c}24.69 \\
(29.8 \pm 0.8)\end{array}$ & $\begin{array}{c}1.44 \\
(1.6 \pm 0.0)\end{array}$ & $\begin{array}{c}55.56 \\
(48.2 \pm 0.0)\end{array}$ & $\begin{array}{c}30.86 \\
(33.7 \pm 0.8)\end{array}$ & $\begin{array}{c}1.44 \\
(1.6 \pm 0.1)\end{array}$ & $\begin{array}{c}55.56 \\
(50.3 \pm 1.1)\end{array}$ \\
\hline \multicolumn{3}{|c|}{$\mathrm{CD}$ at $5 \%$} & 2.53 & 0.09 & 2.89 & 2.43 & 0.10 & 3.53 \\
\hline
\end{tabular}


Table.2 Effect of MS medium with Kinetin \pm NAA/GA 3 on regeneration of hypocotyl explants of Capsicum hybrids Bharat and Indra

\begin{tabular}{|c|c|c|c|c|c|c|c|c|}
\hline \multirow{2}{*}{\multicolumn{3}{|c|}{$\begin{array}{c}\text { MS + growth regulators } \\
(\mathrm{mg} / \mathrm{l})\end{array}$}} & \multicolumn{3}{|c|}{ Bharat } & \multicolumn{3}{|c|}{ Indra } \\
\hline & & & \multirow{2}{*}{$\begin{array}{c}\text { Per cent } \\
\text { Regeneration }\end{array}$} & \multirow{2}{*}{$\begin{array}{l}\text { Elongated shoots } \\
\text { per explant }\end{array}$} & \multirow{2}{*}{$\begin{array}{l}\text { Per cent shoot } \\
\text { elongation }\end{array}$} & \multirow{2}{*}{$\begin{array}{c}\text { Per cent } \\
\text { Regeneration }\end{array}$} & \multirow{2}{*}{$\begin{array}{c}\text { Elongated shoots } \\
\text { per explant }\end{array}$} & \multirow{2}{*}{$\begin{array}{l}\text { Per cent shoot } \\
\text { elongation }\end{array}$} \\
\hline Kinetin & NAA & $\mathbf{G A}_{3}$ & & & & & & \\
\hline 2.5 & 0.0 & 0.0 & $\begin{array}{c}0.00 \\
(0.0 \pm 0.0)\end{array}$ & $\begin{array}{c}0.00 \\
(1.0 \pm 0.0)\end{array}$ & $\begin{array}{c}0.00 \\
(0.0 \pm 0.0)\end{array}$ & $\begin{array}{c}0.00 \\
(0.0 \pm 0.0)\end{array}$ & $\begin{array}{c}0.00 \\
(1.0 \pm 0.0)\end{array}$ & $\begin{array}{c}0.00 \\
(0.0 \pm 0.0)\end{array}$ \\
\hline 2.5 & 0.2 & 0.0 & $\begin{array}{c}0.00 \\
(0.0 \pm 0.0)\end{array}$ & $\begin{array}{c}0.00 \\
(1.0 \pm 0.0)\end{array}$ & $\begin{array}{c}0.00 \\
(0.0 \pm 0.0)\end{array}$ & $\begin{array}{c}0.00 \\
(0.0 \pm 0.0)\end{array}$ & $\begin{array}{c}0.00 \\
(1.0 \pm 0.0)\end{array}$ & $\begin{array}{c}0.00 \\
(0.0 \pm 0.0)\end{array}$ \\
\hline 2.5 & 0.0 & 2.0 & $\begin{array}{c}0.00 \\
(0.0 \pm 0.0) \\
\end{array}$ & $\begin{array}{c}0.00 \\
(1.0 \pm 0.0) \\
\end{array}$ & $\begin{array}{c}0.00 \\
(0.0 \pm 0.0) \\
\end{array}$ & $\begin{array}{c}3.70 \\
(11.1 \pm 0.0) \\
\end{array}$ & $\begin{array}{c}0.00 \\
(1.0 \pm 0.0) \\
\end{array}$ & $\begin{array}{c}0.00 \\
(0.0 \pm 0.0) \\
\end{array}$ \\
\hline 5.0 & 0.0 & 0.0 & $\begin{array}{c}13.58 \\
(21.6 \pm 1.1)\end{array}$ & $\begin{array}{c}0.22 \\
(1.1 \pm 0.1) \\
\end{array}$ & $\begin{array}{c}18.52 \\
(25.4 \pm 1.3) \\
\end{array}$ & $\begin{array}{c}18.52 \\
(25.5 \pm 0.0)\end{array}$ & $\begin{array}{c}0.44 \\
(1.2 \pm 0.1) \\
\end{array}$ & $\begin{array}{c}22.22 \\
(28.1 \pm 0.0) \\
\end{array}$ \\
\hline 5.0 & 0.2 & 0.0 & $\begin{array}{c}6.17 \\
(14.2 \pm 1.6) \\
\end{array}$ & $\begin{array}{c}0.00 \\
(1.0 \pm 0.0) \\
\end{array}$ & $\begin{array}{c}0.00 \\
(0.0 \pm 0.0) \\
\end{array}$ & $\begin{array}{c}9.88 \\
(18.2 \pm 1.2) \\
\end{array}$ & $\begin{array}{c}0.00 \\
(1.0 \pm 0.0) \\
\end{array}$ & $\begin{array}{c}0.00 \\
(0.0 \pm 0.0) \\
\end{array}$ \\
\hline 5.0 & 0.0 & 2.0 & $\begin{array}{c}13.58 \\
(21.6 \pm 1.1)\end{array}$ & $\begin{array}{c}0.67 \\
(1.3 \pm 0.0)\end{array}$ & $\begin{array}{c}38.89 \\
(38.5 \pm 3.3)\end{array}$ & $\begin{array}{c}19.75 \\
(26.4 \pm 0.9)\end{array}$ & $\begin{array}{c}0.78 \\
(1.4 \pm 0.0)\end{array}$ & $\begin{array}{c}48.15 \\
(43.9 \pm 1.1)\end{array}$ \\
\hline 7.5 & 0.0 & 0.0 & $\begin{array}{c}23.46 \\
(28.9 \pm 0.8)\end{array}$ & $\begin{array}{c}1.00 \\
(1.4 \pm 0.0)\end{array}$ & $\begin{array}{c}48.15 \\
(43.9 \pm 1.1)\end{array}$ & $\begin{array}{c}37.04 \\
(37.5 \pm 0.0)\end{array}$ & $\begin{array}{c}1.00 \\
(1.4 \pm 0.0)\end{array}$ & $\begin{array}{c}46.30 \\
(42.8 \pm 2.1)\end{array}$ \\
\hline 7.5 & 0.2 & 0.0 & $\begin{array}{c}11.11 \\
(19.5 \pm 0.0)\end{array}$ & $\begin{array}{c}0.44 \\
(1.2 \pm 0.1)\end{array}$ & $\begin{array}{c}33.33 \\
(35.3 \pm 0.0)\end{array}$ & $\begin{array}{c}18.52 \\
(25.5 \pm 0.0)\end{array}$ & $\begin{array}{c}0.56 \\
(1.3 \pm 0.1)\end{array}$ & $\begin{array}{c}27.78 \\
(31.7 \pm 2.1)\end{array}$ \\
\hline 7.5 & 0.0 & 2.0 & $\begin{array}{c}24.69 \\
(29.8 \pm 0.8)\end{array}$ & $\begin{array}{c}1.44 \\
(1.6 \pm 0.0)\end{array}$ & $\begin{array}{c}59.26 \\
(50.3 \pm 1.1)\end{array}$ & $\begin{array}{c}38.27 \\
(38.2 \pm 0.7)\end{array}$ & $\begin{array}{c}1.78 \\
(1.7 \pm 0.0)\end{array}$ & $\begin{array}{c}62.04 \\
(52.0 \pm 0.6)\end{array}$ \\
\hline 10.0 & 0.0 & 0.0 & $\begin{array}{c}27.16 \\
(31.4 \pm 0.8)\end{array}$ & $\begin{array}{c}1.00 \\
(1.4 \pm 0.0)\end{array}$ & $\begin{array}{c}50.00 \\
(45.0 \pm 1.8) \\
\end{array}$ & $\begin{array}{c}37.04 \\
(37.5 \pm 0.0)\end{array}$ & $\begin{array}{c}1.00 \\
(1.4 \pm 0.0)\end{array}$ & $\begin{array}{c}46.30 \\
(42.8 \pm 2.1) \\
\end{array}$ \\
\hline 10.0 & 0.2 & 0.0 & $\begin{array}{c}12.35 \\
(20.5 \pm 1.1) \\
\end{array}$ & $\begin{array}{c}0.44 \\
(1.2 \pm 0.1) \\
\end{array}$ & $\begin{array}{c}33.33 \\
(35.3 \pm 0.0)\end{array}$ & $\begin{array}{c}17.28 \\
(24.5 \pm 1.0)\end{array}$ & $\begin{array}{c}0.56 \\
(1.3 \pm 0.1) \\
\end{array}$ & $\begin{array}{c}27.78 \\
(31.8 \pm 0.0) \\
\end{array}$ \\
\hline 10.0 & 0.0 & 2.0 & $\begin{array}{c}27.16 \\
(31.8 \pm 0.8) \\
\end{array}$ & $\begin{array}{c}1.56 \\
(1.6 \pm 0.0) \\
\end{array}$ & $\begin{array}{c}61.11 \\
(51.4 \pm 0.0)\end{array}$ & $\begin{array}{c}37.04 \\
(37.5 \pm 0.0) \\
\end{array}$ & $\begin{array}{c}1.78 \\
(1.7 \pm 0.0) \\
\end{array}$ & $\begin{array}{c}61.11 \\
(51.4 \pm 0.0) \\
\end{array}$ \\
\hline \multicolumn{3}{|c|}{$\mathrm{CD}$ at $5 \%$} & 2.46 & 0.08 & 3.60 & 1.63 & 0.09 & 3.26 \\
\hline
\end{tabular}


Table.3 Effect of MS medium with TDZ \pm NAA/GA 3 on regeneration of hypocotyl explants of Capsicum hybrids Bharat and Indra

\begin{tabular}{|c|c|c|c|c|c|c|c|c|}
\hline \multirow{2}{*}{\multicolumn{3}{|c|}{$\begin{array}{c}\text { MS + growth regulators } \\
(\mathrm{mg} / \mathrm{l})\end{array}$}} & \multicolumn{3}{|c|}{ Bharat } & \multicolumn{3}{|c|}{ Indra } \\
\hline & & & \multirow{2}{*}{$\begin{array}{c}\text { Per cent } \\
\text { Regeneration }\end{array}$} & \multirow{2}{*}{$\begin{array}{l}\text { Elongated shoots } \\
\text { per explant }\end{array}$} & \multirow{2}{*}{$\begin{array}{l}\text { Per cent shoot } \\
\text { elongation }\end{array}$} & \multirow{2}{*}{$\begin{array}{c}\text { Per cent } \\
\text { Regeneration }\end{array}$} & \multirow{2}{*}{$\begin{array}{c}\text { Elongated shoots } \\
\text { per explant }\end{array}$} & \multirow{2}{*}{$\begin{array}{l}\text { Per cent shoot } \\
\text { elongation }\end{array}$} \\
\hline TDZ & NAA & $\mathbf{G A}_{3}$ & & & & & & \\
\hline 2.5 & 0.0 & 0.0 & $\begin{array}{c}7.41 \\
(15.79 \pm 0.0)\end{array}$ & $\begin{array}{c}0.00 \\
(1.0 \pm 0.0)\end{array}$ & $\begin{array}{c}33.33 \\
(35.3 \pm 0.0)\end{array}$ & $\begin{array}{c}9.88 \\
(18.2 \pm 1.2)\end{array}$ & $\begin{array}{c}0.00 \\
(1.0 \pm 0.0)\end{array}$ & $\begin{array}{c}0.00 \\
(0.0 \pm 0.0)\end{array}$ \\
\hline 2.5 & 0.2 & 0.0 & $\begin{array}{c}3.70 \\
(11.09 \pm 0.0)\end{array}$ & $\begin{array}{c}0.00 \\
(1.0 \pm 0.0)\end{array}$ & $\begin{array}{c}0.00 \\
(0.0 \pm 0.0)\end{array}$ & $\begin{array}{c}0.00 \\
(0.0 \pm 0.0)\end{array}$ & $\begin{array}{c}0.00 \\
(1.0 \pm 0.0)\end{array}$ & $\begin{array}{c}0.00 \\
(0.0 \pm 0.0)\end{array}$ \\
\hline 2.5 & 0.0 & 2.0 & $\begin{array}{c}7.41 \\
(15.79 \pm 0.0) \\
\end{array}$ & $\begin{array}{c}0.44 \\
(1.2 \pm 0.1) \\
\end{array}$ & $\begin{array}{c}44.44 \\
(41.7 \pm 3.3) \\
\end{array}$ & $\begin{array}{c}11.11 \\
(19.5 \pm 0.0)\end{array}$ & $\begin{array}{c}0.33 \\
(1.2 \pm 0.0) \\
\end{array}$ & $\begin{array}{c}29.63 \\
(32.9 \pm 2.4) \\
\end{array}$ \\
\hline 5.0 & 0.0 & 0.0 & $\begin{array}{c}28.40 \\
(32.2 \pm 0.8) \\
\end{array}$ & $\begin{array}{c}0.33 \\
(1.2 \pm 0.0) \\
\end{array}$ & $\begin{array}{c}42.59 \\
(40.7 \pm 1.1) \\
\end{array}$ & $\begin{array}{c}27.16 \\
(31.4 \pm 0.8) \\
\end{array}$ & $\begin{array}{c}0.22 \\
(1.1 \pm 0.1) \\
\end{array}$ & $\begin{array}{c}22.22 \\
(28.1 \pm 0.0) \\
\end{array}$ \\
\hline 5.0 & 0.2 & 0.0 & $\begin{array}{c}9.88 \\
(18.2 \pm 1.2) \\
\end{array}$ & $\begin{array}{c}0.00 \\
(1.0 \pm 0.0) \\
\end{array}$ & $\begin{array}{c}33.33 \\
(35.3 \pm 0.0) \\
\end{array}$ & $\begin{array}{c}8.64 \\
(17.0 \pm 1.2) \\
\end{array}$ & $\begin{array}{c}0.00 \\
(1.0 \pm 0.0) \\
\end{array}$ & $\begin{array}{c}0.00 \\
(0.0 \pm 0.0) \\
\end{array}$ \\
\hline 5.0 & 0.0 & 2.0 & $\begin{array}{c}30.86 \\
(33.7 \pm 0.8)\end{array}$ & $\begin{array}{c}1.11 \\
(1.5 \pm 0.0)\end{array}$ & $\begin{array}{c}55.56 \\
(48.2 \pm 0.0)\end{array}$ & $\begin{array}{c}29.63 \\
(33.0 \pm 0.0)\end{array}$ & $\begin{array}{c}0.89 \\
(1.4 \pm 0.0)\end{array}$ & $\begin{array}{c}46.30 \\
(42.8 \pm 2.1)\end{array}$ \\
\hline 7.5 & 0.0 & 0.0 & $\begin{array}{c}8.64 \\
(17.0 \pm 1.2)\end{array}$ & $\begin{array}{c}0.00 \\
(1.0 \pm 0.0)\end{array}$ & $\begin{array}{c}16.67 \\
(24.1 \pm 0.0)\end{array}$ & $\begin{array}{c}6.17 \\
(14.2 \pm 1.6)\end{array}$ & $\begin{array}{c}0.00 \\
(1.0 \pm 0.0)\end{array}$ & $\begin{array}{c}0.00 \\
(0.0 \pm 0.0)\end{array}$ \\
\hline 7.5 & 0.2 & 0.0 & $\begin{array}{c}3.70 \\
(11.1 \pm 0.0)\end{array}$ & $\begin{array}{c}0.00 \\
(1.0 \pm 0.0)\end{array}$ & $\begin{array}{c}0.00 \\
(0.0 \pm 0.0)\end{array}$ & $\begin{array}{c}3.70 \\
(11.1 \pm 0.0)\end{array}$ & $\begin{array}{c}0.00 \\
(1.0 \pm 0.0)\end{array}$ & $\begin{array}{c}0.00 \\
(0.0 \pm 0.0)\end{array}$ \\
\hline 7.5 & 0.0 & 2.0 & $\begin{array}{c}8.64 \\
(17.0 \pm 1.2)\end{array}$ & $\begin{array}{c}0.00 \\
(1.0 \pm 0.0)\end{array}$ & $\begin{array}{c}33.33 \\
(35.3 \pm 0.0)\end{array}$ & $\begin{array}{c}7.41 \\
(15.8 \pm 0.0)\end{array}$ & $\begin{array}{c}0.00 \\
(1.0 \pm 0.0)\end{array}$ & $\begin{array}{c}0.00 \\
(0.0 \pm 0.0)\end{array}$ \\
\hline 10.0 & 0.0 & 0.0 & $\begin{array}{c}0.00 \\
(0.0 \pm 0.0)\end{array}$ & $\begin{array}{c}0.00 \\
(1.0 \pm 0.0)\end{array}$ & $\begin{array}{c}0.00 \\
(0.0 \pm 0.0)\end{array}$ & $\begin{array}{c}0.00 \\
(0.0 \pm 0.0)\end{array}$ & $\begin{array}{c}0.00 \\
(1.0 \pm 0.0)\end{array}$ & $\begin{array}{c}0.00 \\
(0.0 \pm 0.0)\end{array}$ \\
\hline 10.0 & 0.2 & 0.0 & $\begin{array}{c}0.00 \\
(0.0 \pm 0.0) \\
\end{array}$ & $\begin{array}{c}0.00 \\
(1.0 \pm 0.0) \\
\end{array}$ & $\begin{array}{c}0.00 \\
(0.0 \pm 0.0) \\
\end{array}$ & $\begin{array}{c}0.00 \\
(0.0 \pm 0.0) \\
\end{array}$ & $\begin{array}{c}0.00 \\
(1.0 \pm 0.0) \\
\end{array}$ & $\begin{array}{c}0.00 \\
(0.0 \pm 0.0) \\
\end{array}$ \\
\hline 10.0 & 0.0 & 2.0 & $\begin{array}{c}0.00 \\
(0.0 \pm 0.0) \\
\end{array}$ & $\begin{array}{c}0.00 \\
(1.0 \pm 0.0) \\
\end{array}$ & $\begin{array}{c}0.00 \\
(0.0 \pm 0.0) \\
\end{array}$ & $\begin{array}{c}0.00 \\
(0.0 \pm 0.0) \\
\end{array}$ & $\begin{array}{c}0.00 \\
(1.0 \pm 0.0)\end{array}$ & $\begin{array}{c}0.00 \\
(0.0 \pm 0.0) \\
\end{array}$ \\
\hline \multicolumn{3}{|c|}{$\mathrm{CD}$ at $5 \%$} & 2.02 & 0.05 & 2.90 & 2.09 & 0.06 & 1.81 \\
\hline
\end{tabular}


Table.4 Effect of MS medium with Zeatin \pm NAA/GA 3 on regeneration of hypocotyl explants of Capsicum hybrids Bharat and Indra

\begin{tabular}{|c|c|c|c|c|c|c|c|c|}
\hline \multirow{2}{*}{\multicolumn{3}{|c|}{$\begin{array}{c}\text { MS + growth regulators } \\
(\mathrm{mg} / \mathrm{l})\end{array}$}} & \multicolumn{3}{|c|}{ Bharat } & \multicolumn{3}{|c|}{ Indra } \\
\hline & & & \multirow{2}{*}{$\begin{array}{c}\text { Per cent } \\
\text { Regeneration }\end{array}$} & \multirow{2}{*}{$\begin{array}{l}\text { Elongated shoots } \\
\text { per explant }\end{array}$} & \multirow{2}{*}{$\begin{array}{l}\text { Per cent shoot } \\
\text { elongation }\end{array}$} & \multirow{2}{*}{$\begin{array}{c}\text { Per cent } \\
\text { Regeneration }\end{array}$} & \multirow{2}{*}{$\begin{array}{c}\text { Elongated shoots } \\
\text { per explant }\end{array}$} & \multirow{2}{*}{$\begin{array}{l}\text { Per cent shoot } \\
\text { elongation }\end{array}$} \\
\hline Zeatin & NAA & $\mathbf{G A}_{3}$ & & & & & & \\
\hline 2.5 & 0.0 & 0.0 & $\begin{array}{c}4.94 \\
(12.7 \pm 1.6)\end{array}$ & $\begin{array}{c}0.00 \\
(1.0 \pm 0.0)\end{array}$ & $\begin{array}{c}0.00 \\
(0.0 \pm 0.0)\end{array}$ & $\begin{array}{c}7.41 \\
(15.8 \pm 0.0)\end{array}$ & $\begin{array}{c}0.00 \\
(1.0 \pm 0.0)\end{array}$ & $\begin{array}{c}0.00 \\
(0.0 \pm 0.0)\end{array}$ \\
\hline 2.5 & 0.2 & 0.0 & $\begin{array}{c}0.00 \\
(0.0 \pm 0.0)\end{array}$ & $\begin{array}{c}0.00 \\
(1.0 \pm 0.0)\end{array}$ & $\begin{array}{c}0.00 \\
(0.0 \pm 0.0)\end{array}$ & $\begin{array}{c}0.00 \\
(0.0 \pm 0.0)\end{array}$ & $\begin{array}{c}0.00 \\
(1.0 \pm 0.0)\end{array}$ & $\begin{array}{c}0.00 \\
(0.0 \pm 0.0)\end{array}$ \\
\hline 2.5 & 0.0 & 2.0 & $\begin{array}{c}4.94 \\
(12.7 \pm 1.6)\end{array}$ & $\begin{array}{c}0.22 \\
(1.1 \pm 0.1)\end{array}$ & $\begin{array}{c}0.00 \\
(0.0 \pm 0.0)\end{array}$ & $\begin{array}{c}7.41 \\
(15.8 \pm 0.0)\end{array}$ & $\begin{array}{c}0.44 \\
(1.2 \pm 0.1) \\
\end{array}$ & $\begin{array}{c}16.67 \\
(24.1 \pm 0.0)\end{array}$ \\
\hline 5.0 & 0.0 & 0.0 & $\begin{array}{c}17.28 \\
(24.5 \pm 1.0)\end{array}$ & $\begin{array}{c}0.44 \\
(1.2 \pm 0.1)\end{array}$ & $\begin{array}{c}33.33 \\
(35.3 \pm 0.0)\end{array}$ & $\begin{array}{c}22.22 \\
(28.1 \pm 0.0)\end{array}$ & $\begin{array}{c}0.56 \\
(1.3 \pm 0.1)\end{array}$ & $\begin{array}{c}29.63 \\
(33.0 \pm 1.2)\end{array}$ \\
\hline 5.0 & 0.2 & 0.0 & $\begin{array}{c}8.64 \\
(17.0 \pm 1.2)\end{array}$ & $\begin{array}{c}0.11 \\
(1.1 \pm 0.1)\end{array}$ & $\begin{array}{c}16.67 \\
(24.1 \pm 0.0)\end{array}$ & $\begin{array}{c}12.35 \\
(20.5 \pm 1.1)\end{array}$ & $\begin{array}{c}0.11 \\
(1.1 \pm 0.1)\end{array}$ & $\begin{array}{c}18.52 \\
(25.4 \pm 1.3)\end{array}$ \\
\hline 5.0 & 0.0 & 2.0 & $\begin{array}{c}17.28 \\
(24.5 \pm 1.0)\end{array}$ & $\begin{array}{c}0.89 \\
(1.4 \pm 0.0)\end{array}$ & $\begin{array}{c}38.89 \\
(38.5 \pm 3.3)\end{array}$ & $\begin{array}{c}22.22 \\
(28.11 \pm 0.03)\end{array}$ & $\begin{array}{c}1.11 \\
(1.5 \pm 0.0)\end{array}$ & $\begin{array}{c}51.85 \\
(46.1 \pm 1.1)\end{array}$ \\
\hline 7.5 & 0.0 & 0.0 & $\begin{array}{c}30.86 \\
(33.7 \pm 0.8)\end{array}$ & $\begin{array}{c}1.00 \\
(1.4 \pm 0.0)\end{array}$ & $\begin{array}{c}51.85 \\
(46.1 \pm 1.1)\end{array}$ & $\begin{array}{c}43.21 \\
(41.1 \pm 0.7)\end{array}$ & $\begin{array}{c}1.33 \\
(1.5 \pm 0.0)\end{array}$ & $\begin{array}{c}48.89 \\
(44.3 \pm 1.3)\end{array}$ \\
\hline 7.5 & 0.2 & 0.0 & $\begin{array}{c}13.58 \\
(21.6 \pm 1.1)\end{array}$ & $\begin{array}{c}0.56 \\
(1.3 \pm 0.1)\end{array}$ & $\begin{array}{c}33.33 \\
(35.2 \pm 0.0)\end{array}$ & $\begin{array}{c}18.52 \\
(25.5 \pm 0.0)\end{array}$ & $\begin{array}{c}0.67 \\
(1.3 \pm 0.0)\end{array}$ & $\begin{array}{c}35.19 \\
(36.3 \pm 2.9)\end{array}$ \\
\hline 7.5 & 0.0 & 2.0 & $\begin{array}{c}32.10 \\
(34.5 \pm 0.8)\end{array}$ & $\begin{array}{c}1.67 \\
(1.6 \pm 0.0)\end{array}$ & $\begin{array}{c}61.11 \\
(51.4 \pm 1.9)\end{array}$ & $\begin{array}{c}44.44 \\
(41.8 \pm 1.2)\end{array}$ & $\begin{array}{c}2.11 \\
(1.8 \pm 0.0)\end{array}$ & $\begin{array}{c}66.85 \\
(54.8 \pm 1.1)\end{array}$ \\
\hline 10.0 & 0.0 & 0.0 & $\begin{array}{c}34.57 \\
(36.0 \pm 0.7)\end{array}$ & $\begin{array}{c}1.11 \\
(1.5 \pm 0.0)\end{array}$ & $\begin{array}{c}53.70 \\
(47.1 \pm 1.1)\end{array}$ & $\begin{array}{c}43.21 \\
(41.1 \pm 0.7)\end{array}$ & $\begin{array}{c}1.33 \\
(1.5 \pm 0.0)\end{array}$ & $\begin{array}{c}49.07 \\
(44.5 \pm 0.5)\end{array}$ \\
\hline 10.0 & 0.2 & 0.0 & $\begin{array}{c}14.81 \\
(22.6 \pm 0.0)\end{array}$ & $\begin{array}{c}0.56 \\
(1.3 \pm 0.1)\end{array}$ & $\begin{array}{c}38.89 \\
(38.5 \pm 3.3)\end{array}$ & $\begin{array}{c}18.52 \\
(25.0 \pm 0.0)\end{array}$ & $\begin{array}{c}0.67 \\
(1.3 \pm 0.0)\end{array}$ & $\begin{array}{c}35.19 \\
(36.4 \pm 1.1)\end{array}$ \\
\hline 10.0 & 0.0 & 2.0 & $\begin{array}{c}34.57 \\
(36.0 \pm 0.7)\end{array}$ & $\begin{array}{c}1.78 \\
(1.7 \pm 0.0)\end{array}$ & $\begin{array}{c}64.81 \\
(53.6 \pm 1.1)\end{array}$ & $\begin{array}{c}43.21 \\
(41.1 \pm 0.7)\end{array}$ & $\begin{array}{c}2.11 \\
(1.8 \pm 0.0)\end{array}$ & $\begin{array}{c}66.67 \\
(54.7 \pm 0.0)\end{array}$ \\
\hline \multicolumn{3}{|c|}{$\mathrm{CD}$ at $5 \%$} & 2.89 & 0.11 & 4.49 & 1.73 & 0.10 & 3.52 \\
\hline
\end{tabular}


Table.5 Effect of MS medium supplemented with different auxins on rooting of in vitro induced shoots from Hypocotyl explants of both the capsicum hybrids

\begin{tabular}{|c|c|c|c|c|c|c|c|c|}
\hline \multirow{2}{*}{\multicolumn{2}{|c|}{$\begin{array}{c}\text { MS } \\
\text { medium } \\
\text { with } \\
\text { auxins } \\
(\mathrm{mg} / \mathrm{l})\end{array}$}} & \multicolumn{6}{|c|}{ Genotype } & \multirow{3}{*}{$\begin{array}{c}\text { Types of roots } \\
\text { formed on induced } \\
\text { shoots }\end{array}$} \\
\hline & & \multicolumn{3}{|c|}{ Bharat } & \multicolumn{3}{|c|}{ Indra } & \\
\hline IBA & NAA & $\begin{array}{c}\text { Per } \\
\text { Cent rooting* }\end{array}$ & $\begin{array}{c}\text { Roots } \\
\text { per shoot }\end{array}$ & $\begin{array}{c}\text { Root length } \\
(\mathrm{cm})\end{array}$ & $\begin{array}{c}\text { Per } \\
\text { Cent rooting* }\end{array}$ & $\begin{array}{c}\text { Roots } \\
\text { Per shoot }\end{array}$ & $\begin{array}{c}\text { Root } \\
\text { length }(\mathrm{cm})\end{array}$ & \\
\hline 0.0 & 0.0 & $4.44(5.9 \pm 3.9)$ & $7.07 \pm 0.42$ & $8.51 \pm 0.12$ & $11.11(14.8 \pm 4.7)$ & $2.44 \pm 0.18$ & $7.30 \pm 0.08$ & Long and thin roots \\
\hline 0.5 & 0.0 & $\begin{array}{c}100 \\
(90.0 \pm 0.0)\end{array}$ & $23.65 \pm 0.24$ & $7.71 \pm 0.07$ & $100(90.0 \pm 0.0)$ & $27.56 \pm 0.24$ & $4.94 \pm 0.04$ & $\begin{array}{c}\text { Medium long, thick } \\
\text { roots }\end{array}$ \\
\hline 1.0 & 0.0 & $\begin{array}{c}100 \\
(90.0 \pm 0.0)\end{array}$ & $23.04 \pm 0.19$ & $6.68 \pm 0.07$ & $100(90.0 \pm 0.0)$ & $25.67 \pm 0.41$ & $4.29 \pm 0.04$ & $\begin{array}{l}\text { Short thick roots } \\
\text { with slight callus }\end{array}$ \\
\hline 0.0 & 0.5 & $\begin{array}{c}100 \\
(90.0 \pm 0.0)\end{array}$ & $17.54 \pm 0.31$ & $4.41 \pm 0.05$ & $100(90.0 \pm 0.0)$ & $14.56 \pm 0.34$ & $1.91 \pm 0.06$ & $\begin{array}{l}\text { Short thick roots } \\
\text { with callus }\end{array}$ \\
\hline 0.0 & 1.0 & $\begin{array}{c}51.11 \\
(45.6 \pm 2.0)\end{array}$ & $14.01 \pm 0.32$ & $1.89 \pm 0.05$ & $\begin{array}{c}66.67 \\
(55.0 \pm 2.1)\end{array}$ & $12.44 \pm 0.18$ & $1.12 \pm 0.05$ & $\begin{array}{l}\text { Short thick roots } \\
\text { with callus }\end{array}$ \\
\hline \multicolumn{2}{|c|}{$\mathrm{CD}$ at $5 \%$} & 5.64 & 0.88 & 0.22 & 6.57 & 0.81 & 0.15 & \\
\hline
\end{tabular}

* Figures in parenthesis are angular transformed values 
The better regeneration was observed in chilli genotype G4 compared to LCA334 from cotyledonary and hypocotyl explants on MS medium supplemented with $0.25 \mathrm{mg} / \mathrm{l}$ zeatin and $2 \mathrm{mg} / \mathrm{l}$ phenyl acetic acid (PAA). Among the explants, cotyledonary leaf exhibited a higher regeneration response compared to that of hypocotyls (Manamohan et al., 2016).

Hormones play a crucial role in controlling the plant growth and development. To induce cell division, elongation and growth of plant in tissue cultures; auxins, cytokinins and other are synergistically required. Among the cytokinins tested, zeatin followed by kinetin, was found superior with maximum per cent shoot elongation but minimum was observed in TDZ in both the hybrids. The common problem with TDZ was inhibition of shoot elongation. It might be super-optimal cytokinin activity and the presence of a phenyl group in TDZ (Huetteman and Preece, 1993; Steinitz et al., 2003). In the study, shoot buds induced did not elongate properly from explants on a medium containing TDZ and resulted in a rosette of shoots when continued to be cultured on the same medium. Thus, it needs to culture the explants on medium containing TDZ along with $\mathrm{GA}_{3}$ in order to get elongated shoots. Similar results were observed in the capsicum by Vivek Hegde et al., (2017).

Good regeneration response in both the hybrids was observed on MS medium supplemented with BAP, kinetin and zeatin at 7.5 and $10.0 \mathrm{mg} / \mathrm{l}$ along with $2.0 \mathrm{mg} / \mathrm{l} \mathrm{GA}_{3}$ (Tables 1, 2 and 4) and TDZ at $5.0 \mathrm{mg} / \mathrm{l}$ along with $2.0 \mathrm{mg} / \mathrm{l} \mathrm{GA}_{3}$, among the different concentrations of plant growth regulators tested. The higher concentrations of TDZ lead to death of explants (Table 3). The regeneration medium supplemented with zeatin at $7.5 \mathrm{mg} / \mathrm{l}$ along with $2.0 \mathrm{mg} / \mathrm{l} \mathrm{GA}_{3}$ recorded maximum per cent regeneration $(44.4 \%)$, more number of elongated shoots per explant (2.1) and highest per cent shoot elongation $(66.9 \%)$ in hypocotyl explants of hybrid Indra (Plate 1B and C) and in hybrid Bharat, maximum per cent regeneration (32.1\%), more number of elongated shoots per explant (1.7) and highest per cent shoot elongation $(61.1 \%)$ were observed on same medium (Table 4). Ebida and $\mathrm{Hu}$ (1993) used the higher concentration of BAP at $10.0 \mathrm{mg} / \mathrm{l}$ to obtain multiple shoots from capsicum hypocotyl explant. Nancy et al., (2005) cultured nodal explants of Habanero pepper and observed multiple shoots on MS medium supplemented with $3.4 \mu \mathrm{M}$ TDZ. Sanatombi and Sharma (2008) obtained maximum number of shoots in shoot-tip of Capsicum chinense Jacq. cv. Umorok, on medium containing 91.2 $\mu \mathrm{M}$ BAP and $31.1 \mu \mathrm{M}$ TDZ with $4.7 \mu \mathrm{M}$ Kinetin. Shoot multiplication in four chilli cultivars was obtained in MS medium with $6.0 \mathrm{mg} / \mathrm{l} \mathrm{BAP,} 1.0 \mathrm{mg} / \mathrm{l}$ kinetin and $0.5 \mathrm{mg} / \mathrm{l} \mathrm{GA} \mathrm{G}_{3}$ (Ranjan et al., 2010). Khurana et al., (2011) standardised a protocol of plant regeneration in male sterile line of chilli, MS-12 was regenerated on MS medium supplemented with $9.0 \mathrm{mg} / \mathrm{l}$ BAP, $2.0 \mathrm{mg} / \mathrm{l}$ kinetin and $2.0 \mathrm{mg} / \mathrm{l}$ IAA. Dafadar et al., (2012) obtained the maximum number of shoots per leaf explants of chilli on medium containing BAP at $8.87 \mu \mathrm{M}$ and $2.85 \mu \mathrm{M}$ IAA. Rahul et al., (2015) regenerated Naga chili on MS medium containing $5 \mathrm{mg} / \mathrm{l} \mathrm{BAP}$ and $0.5 \mathrm{mg} / \mathrm{l}$ IAA. Sharma et al., (2017) observed $60 \%$ regeneration and $40 \%$ shoot elongation from cotyledonary leaf explants of Capsicum frutescens cultured on medium containing $44.44 \mu \mathrm{M}$ BA, $5.71 \mu \mathrm{M}$ IAA, 10 $\mu \mathrm{M} \quad \mathrm{AgNO}_{3}$ and $1.98 \mathrm{mg} \quad \mathrm{L}-1 \quad 2-(\mathrm{N}-$ morpholine) ethane sulphonic acid.

Root induction was done by sub-culturing the shoots on MS medium supplemented different rooting hormones. Cent per cent rooting was observed in regenerated shoots of capsicum hybrid Bharat and Indira on MS medium containing IBA at $0.5,1.0 \mathrm{mg} / \mathrm{l}$ and $0.5 \mathrm{mg} / \mathrm{l}$ 
NAA followed by 51.1 per cent and 66.7 per cent with $1.0 \mathrm{mg} / \mathrm{l} \mathrm{NAA}$ in hybrids Bharat and Indra, respectively (Table 5). Medium long, thick roots in optimum number with 100 per cent were observed on basal MS medium supplemented with IBA at 0.5 or $1.0 \mathrm{mg} / \mathrm{l}$. The number of roots per shoot (27.6 and 23.7) with optimum length $(4.9 \mathrm{~cm}$ and $7.7 \mathrm{~cm})$ was observed in both the hybrids Indra and Bharat, respectively (Table 5). Media containing NAA had short and thick roots while these were medium long and thick on media containing IBA (Plate 2 and Table 5). Optimum root induction was reported by Kim et al., (2008) by sub-culturing the capsicum shoots on MS medium with NAA $0.3 \mathrm{mg} / \mathrm{l}$. Kumar et al., (2012) observed 100\% rooting when elongated shoots were transferred to MS medium supplemented with NAA at $0.5 \mathrm{mg} / \mathrm{l}$. Rooting of the chilli shoots were the highest in MS medium with $2 \mathrm{mg} / \mathrm{l}$ IBA compared to other hormones (Manamohan et al., 2016).

Plantlets with well-developed roots were transferred to pots containing sterile mixture of coco-peat and vermiculite (1:1) and kept under poly house for hardening (Plate 3A). Plantlets were transferred to shade net in big size pot containing soil: sand: farmyard manure (1:1:1), after 14 days of acclimatization under poly house conditions (Plate 3B). Per cent survival was $92.31 \%$ and $85.71 \%$ in Indra and Bharat, respectively.

The present investigation proved that in vitro plant regeneration is highly dependent on genotypes and plant growth regulators. Hence, before conducting the transformation experiments, regeneration of virus free plants, experiments on creation of novel somaclonal variation or in vitro selection for abiotic and biotic stresses etc., it becomes necessary to standardize the in vitro regeneration system for the targeted genotypes. Here, a reproducible and efficient regeneration protocol for capsicum hybrids Bharat and Indra was developed, which can be exploited for further research and mass production.

\section{References}

Andreoli, C. and Khan, A A. 1999. Matriconditioning integrated with GA to hasten germination and improve stand establishment of pepper and tomato seeds. Brazilian Journal of Agricultural Research, 34 (10):1953-1959.

Dabauza, M. and Pena, L. 2001. High efficiency organogenesis in sweet pepper (Capsicum annuum L.) tissues from different seedling explants. Plant Growth Regulation, 33: 221-229.

Dafadar, A., Das, A., Bandyopadhyay, S. and Jha, T. B. 2012. In vitro propagation and molecular evaluation of a Capsicum annuum L. cultivar with a high chromosome number $(2 \mathrm{n}=48)$. Scientia Horticulturae, 140: 119-124.

Ebida, A. I. A. and $\mathrm{Hu}, \mathrm{C} . Y$. 1993. In vitro morphogenetic responses and plant regeneration from pepper (Capsicum annuum L. CV. Early California Wonder) seedling explants. Plant Cell Reports, 13:107-110.

Egea, C., Dickinson, M. J., Candela, M. and Candela, M. E. 2002. $\beta$ - 1, 3-glucanase isoenzyme and genes in resistant and susceptible pepper (C. annuum) cultivars infected with Phytopthora capsici. Physiologia Plantarum, 107: 312-318.

Groot, S.P.C. and Karssen, C.M. 1987. Gibberellins regulate seed germination in tomato by endosperm weakening: a study with gibberellin-deficient mutants. Planta, 171: 525-531.

Huetteman, C. A. and Preece J. E. 1993. Thidiazuron: A potent cytokinin for woody plant tissue culture. Plant Cell Tissue and Organ Culture, 33: 105-119. Khurana, D.S., Kannanghara, K.N., Devi, R. 
and Gosal, S.S. 2011. In vitro clonal propagation of a male sterile line in chilli crop. Vegetable Science, 38(2): 123-127.

Kim, M., Jang, I.C., Kim, J.A., Park, E.J., Yoon, M. and Lee, Y. 2008. Embryogenesis and plant regeneration of hot pepper (Capsicum annuum L.) through isolated microspore culture. Plant Cell Reports, 27:425-434.

Kumar, R.V., Sharma, V. K., Chattopadhyay, B. and Chakraborty, S. 2012. An improved plant regeneration and Agrobacterium-mediated transformation of red pepper (Capsicum annuum L.). Physiology and Molecular Biology of Plants, 18(4): 357-364.

Manamohan Maligeppagol, Manjula R., Prakash M. N., Prasad Babu K., Bhimanagoud Kumbar M. and Laxman R. H. 2016. Genetic transformation of chilli (Capsicum annuum L.) with Dreb1A transcription factor known to impart drought tolerance. Indian Journal of Biotechnology, 15: 17-24.

Marta, O. And Pawel, N. 2015. In vitro plant regeneration of 4 Capsicum spp. genotypes using different explant types. Turkish Journal of Biology, 39: 60-68.

Mathew, D. 2002. In vitro shoot and root morphogenesis from cotyledon and hypocotyls explants of hot pepper cultivars Byadgi Dabbi and Arka Lohit. Capsicum and Eggplant Newsletter, 21: 69-72.

Nancy, S.B., Adriana, C.F., Felipe, B.P., Maria, C.M.P., Patricia, Y.Z.C., Anabel, S. R., Amilcar, Z.C., Omar, G.A. and Maria, L.M.H. 2005. Regeneration of Habanero pepper (Capsicum chinense Jacq.) via organogenesis. HortScience, 40(6):1829-1831.

Ochoa, N.A. and, Ramirez, M.R. 2001. In vitro chilli pepper biotechnology. In Vitro Cellular and Developmental Biology -Plant, 37:701-729.
Rahul P. Raj, Glint V. D. and Nirmal Babu K. 2015. In vitro plant regeneration in Capsicum chinense Jacq. (Naga Chili). Journal of Applied Biology and Biotechnology, 3 (01): 030-033.

Ranjan, J.K., Singh, S.K., Chakrabarti, A.K. and Pragya, 2010. In vitro shoot regeneration from cotyledonary leaf explants in chilli and bio-hardening of plantlets. Indian Journal of Horticulture, 67(1): 43-49.

Sanatombi, K. and Sharma, G.J. 2008. In vitro propagation of Capsicum chinense Jacq. Biologia Plantarum, 52 (3): 517-520.

Sharma Ashwani, Gokare Aswathanarayana Ravishankar, Parvatam Giridhar 2017. Silver nitrate and 2-(N-morpholine) ethane sulphonic acid in culture medium promotes rapid shoot regeneration from the proximal zone of the leaf of Capsicum frutescens Mill. Plant Cell Tissue and Organ Culture, 129:175-180.

Simonne, A. H., Simonne, E. H., Eitenmiller, R. R., Mills, H. A. and Green, N. R. 1997. Ascorbic acid and provitamin A contents in unusually colored bell peppers (Capsicum annuum L.). Food Components Analysis, 10: 299-311.

Steinitz, B., Kusek, M., Tabib, Y., Paran, I. and Zelcer, A. 2003. Pepper (Capsicum annuиm L.) regenerants obtained by direct somatic embryogenesis fail to develop a shoot. In Vitro Cellular and Developmental Biology -Plant, 39: 296303.

Suzuki, K. and Mori, M. 2003. Carotenoid composition of new cultivar of Capsicum annuum during maturation and its high capsanthin content. Journal of Japanese Society of Food Science and Technology, 50: 324-326.

Valadez, B.M.G., Aguado S.G.A., Carrillo, C.G., Aguilar, R. V. H., Espitia, R. E., Montes, H. S. and Robledo, P. A. 2009. In vitro propagation and agronomic 
performance of regenerated chilli pepper (Capsicum spp.) plants from commercially important genotypes. In Vitro Cellular and Developmental Biology - Plant, 45:650-658.

Venkataiah. P., Christopher, T. and Subhash, K. 2003. Thiadiazuron induced high frequency adventitious shoot formation and plant regeneration in Capsicum annuum L. Journal of Plant Biotechnology, 5: 245-250.

Vivek Hegde, P.S. Partap and R.C. Yadav 2017. In Vitro Regeneration of
Capsicum (Capsicum annuum L.) from Cotyledon Explants. International Journal of Current Microbiology and Applied Sciences, 6(5): 225-237.

Watkins, J. T. and Cantlife, J. D. 1983. Hormonal control of pepper seed germination. HortScience, 18 (3): 342343.

Watkins, J. T., Cantlife, J. D., Huber, D.J. and Nell T.A. 1985. Gibberellic acid stimulated degradation of endosperm in pepper. Journal of the American Society for Horticultural Science, 110: 61-65.

\section{How to cite this article:}

Vivek Hegde, P.S. Partap and Yadav, R.C. 2017. Plant Regeneration from Hypocotyl Explants in Capsicum (Capsicum Annuum L.). Int.J.Curr.Microbiol.App.Sci. 6(7): 545-557. doi: https://doi.org/10.20546/ijcmas.2017.607.066 